Trauma Berufskrankh 2008 · 10 [Suppl 3]:281-282

DOI 10.1007/s10039-007-1326-0

Online publiziert: 15. Dezember 2007

(c) Springer Medizin Verlag 2007

B. Fellmer Landesverband Südwestdeutschland der gewerblichen Berufsgenossenschaften, Heidelberg

\title{
Begrüßung und Eröffnung der 32. Unfallmedizinischen Tagung am 20./21.10.2007 in Baden-Baden
}

Im Namen des Landesverbandes Südwestdeutschland der gewerblichen Berufsgenossenschaften heiße ich Sie hier im Kurhaus Baden-Baden zur 32. Unfallmedizinischen Tagung herzlich willkommen. Ich begrüße auch sehr herzlich die wissenschaftlichen Leiter der diesjährigen Tagung, die Herren Professor Weise und Professor Wentzensen, die ein prall gefülltes Tagungsprogramm mit hochinteressanten Themen erstellt haben. Ihnen ist das Kunststück gelungen, ein Programm zusammenzustellen, das sowohl dem Interesse der Kliniker als auch dem der niedergelassenen Ärzte als auch dem der Verwaltungsmitarbeiter Rechnung trägt.

Sitzungsvorsitzende, Berichterstatter und Referenten von Rang und Namen haben es auch in diesem Jahr übernommen, die Tagung zu gestalten. Sie sind Garanten für deren Erfolg und haben ohne Zögern ihre Zusage gegeben und die zusätzliche Belastung im Zusammenhang mit der Vorbereitung auf ihren Einsatz bei unserer Tagung auf sich genommen. Hierfür gilt ihnen mein besonderer Dank.

Ich freue mich, dass auch in diesem Jahr wieder eine so große Zahl an Teilnehmern den Weg in das herbstliche Baden-Baden gefunden hat. Neben Ihrem Interesse für unfallchirurgische und Verwaltungsthemen bekunden Sie damit auch Ihre Verbundenheit zur gesetzlichen Unfallversicherung, zu unserem Landesverband. Zudem sehe ich hierin auch den Willen zur verstärkten $\mathrm{Zu}$ sammenarbeit als eine wichtige Voraussetzung für den Erhalt und die Fortentwicklung des unfallmedizinischen Qualitäts- standards. Sie, die Ärztinnen und Ärzte, brauchen wir dabei. Wir sind auf Sie angewiesen, denn Sie sind die tragende Säule der medizinischen Rehabilitation. Unsere Unfallmedizinische Tagung ist deshalb v. a. eine Veranstaltung, die sich an Sie richtet.

Vor dem Hintergrund eines scheinbaren Spannungsfeldes von Qualität und Kosten haben Veranstaltungen wie die Unfallmedizinischen Tagungen der Landesverbände besondere Bedeutung. Sie bilden ein Forum, um wissenschaftliche Erkenntnisse und praktische Erfahrungen zur Diskussion zu stellen und Lösungsmöglichkeiten für die Praxis aufzuzeigen. Sie vermitteln Ihnen Informationen für die Zusammenarbeit zwischen Ärzten und Unfallversicherungsträgern und zeigen Wege auf, wie wir diese Zusammenarbeit effizienter gestalten können.

Der gesetzlichen Unfallversicherung und somit auch den Landesverbänden der gewerblichen Berufsgenossenschaften stehen z. T. einschneidende Änderungen bevor. Der Geschäftsführer des Landesverbandes, Herr Dr. Radek, wird hierüber berichten. Auch wenn wir noch nicht genau wissen, wie sich die Entwicklung für die Landesverbände konkret gestalten wird - in einem sind wir sehr optimistisch: Wir gehen davon aus, dass es auch in Zukunft Unfallmedizinische Tagungen geben wird; dokumentiert haben wir unseren Optimismus darin, dass wir schon jetzt Termine für 2009 und 2011, hier im Kurhaus, vereinbart haben.

Ihnen allen wünsche ich, dass Sie trotz des umfangreichen Tagungsprogramms 
auch Zeit finden werden, hier in BadenBaden einige anregende und schöne Stunden zu verbringen. Auch der gesellschaftliche Aspekt soll nicht zu kurz kommen. Mit unserem Rahmenprogramm möchten wir dazu beitragen. Die Nichtkongressteilnehmer sind am heutigen Nachmittag, um 14.3o Uhr, zu einer Spieledemonstration in der Spielbank eingeladen. Ferner findet heute Abend um 20.30 Uhr, hier in diesem wunderschönen Benazét-Saal, unser traditioneller Gesellschaftsabend mit Tanz und einem attraktiven Rahmenprogramm statt. Der Einlass ist ab 20:0o Uhr. Der Eintritt ist frei. Auch hierzu sind Sie alle herzlich eingeladen.

Bevor ich nun die Tagung in die Hände der wissenschaftlichen Leiter lege, möchte ich mich an den Geschäftsführer des Landesverbandes, Herrn Dr. Erwin Radek, wenden: „Denn dies ist die letzte Unfallmedizinische Tagung, an der Sie als Geschäftsführer des Landesverbandes Südwestdeutschland der gewerblichen Berufsgenossenschaften teilnehmen. Sie scheiden mit Erreichen der Altersgrenze Ende des nächsten Monats aus den Diensten des Landesverbandes aus. Damit endet eine nahezu 13-jährige äußerst erfolgreiche Tätigkeit für den Landesverband Südwestdeutschland. Herr Dr. Radek, Sie haben insbesondere durch viele Kontakte, Kooperationen, Initiativen und Projekte maßgeblich dazu beigetragen, Qualität und Rahmenbedingungen für die ärztliche Arbeit zu fördern und zu steigern. Vor allem die Unfallmedizinischen Tagungen waren Ihnen immer ein besonderes Anliegen. Bezeichnend für Ihre besonderen Verdienste auf diesem Gebiet ist die Tatsache, dass Ihnen bei der Unfallmedizinischen Tagung vor 2 Jahren hier an dieser Stelle das Ehrenzeichen der Deutschen Ärzteschaft durch die Bundesärztekammer verliehen wurde. Obwohl die offizielle Verabschiedung noch aussteht, möchte ich Ihnen, Herrn Dr. Radek, sicherlich auch im Auftrag der Teilnehmer dieser Tagung, ganz herzlich für Ihren Einsatz zum Wohle der arbeitsunfallverletzten und berufserkrankten Menschen danken."
Ich eröffne nunmehr die 32. Unfallmedizinische Tagung des Landesverbandes Südwestdeutschland und gebe das Wort weiter an Herrn Professor Weise.

\section{B. Fellmer}

\section{Korrespondenzadresse}

\section{B. Fellmer}

Landesverband Südwestdeutschland der gewerblichen Berufsgenossenschaften Kurfürsten-Anlage 62, 69115 Heidelberg service@heidelberg.Ivbg.de 